Research Paper

\title{
Expression of Human Leukocyte Antigen G is associated with Prognosis in Nasopharyngeal Carcinoma
}

\author{
Man-Bo Cai ${ }^{1,2 *}$, Hui-Qiong Han ${ }^{1 *}$, Jin-Xin Bei ${ }^{* *}$, Chao-Chun Liü, Jin-Ju Lei ${ }^{1}$, Qian Cui ${ }^{1}$, Qi-Sheng Feng ${ }^{1}$, \\ Hai-Yun Wang ${ }^{4}$, Jia-Xing Zhang ${ }^{4}$, Yi Liang ${ }^{1}$, Li-Zhen Chen ${ }^{1}$, Tie-Bang Kang ${ }^{1}$, Jian-Yong Shao ${ }^{4}$, Yi-Xin \\ Zeng $^{1 凶}$
}

1. State Key Laboratory of Oncology in South China, Cancer Center, Sun Yat-Sen University, Guangzhou, China;

2. Cancer Research Institute, University of South China, Hengyang, China;

3. Wadsworth Center, New York State Department of Health, Albany, New York, USA;

4. Department of Molecular Diagnostics, Cancer Center, Sun Yat-Sen University, Guangzhou, China.

* These authors contributed equally to this work.

$\triangle$ Corresponding author: Yi-Xin Zeng, State Key Laboratory of Oncology in South China, Sun Yat-Sen University Cancer Center, 651 Dongfeng Road East, Guangzhou 510060, China. Phone: +86-20-87343333; Fax: +86-20-87343295; E-mail: zengyx@sysucc.org.cn

() Ivyspring International Publisher. This is an open-access article distributed under the terms of the Creative Commons License (http://creativecommons.org/ licenses/by-nc-nd/3.0/). Reproduction is permitted for personal, noncommercial use, provided that the article is in whole, unmodified, and properly cited.

Received: 2012.03.19; Accepted: 2012.06.05; Published: 2012.06.16

\begin{abstract}
Human leukocyte antigen $\mathrm{G}$ (HLA-G) has multiple immune regulatory functions including the induction of immune tolerance in malignancies. The roles of HLA-G have not been investigated in nasopharyngeal carcinoma (NPC). This study is aimed to evaluate the role of HLA-G as prognostic factor for NPC patients as well as its role in the immune regulation. Western assays showed high HLA-G expression in NPC cell lines, but low in the immortalized nasopharyngeal epithelial cell line NP69. HLA-G protein was further detected in $79.2 \%$ of 552 NPC specimens with immunohistochemistry (IHC), but not in normal nasopharyngeal epithelium tissue. Moreover, high expression of HLA-G predicted poor survival of NPC patients and positively correlated with tumor $\mathrm{N}$ classification and recurrence or metastasis. Multivariate analysis indicated that HLA-G was an independent and unfavorable prognostic factor. Furthermore, the presence of $\mathrm{CD} 68^{+}$macrophages and IL- 10 were also examined, which are two prognostic markers of NPC and important factors for regulating immune surveillance. The correlations of HLA-G with these two immune factors were revealed in NPC tissues. Taken together, our results suggest that HLA-G is an independent biomarker for NPC prognosis, and HLA-G might contribute to NPC progression, which might jointly regulate immune surveillance in NPC together with macrophages and IL-I0.
\end{abstract}

Key words: Nasopharyngeal carcinoma, HLA-G, Prognosis, IL-10, macrophage

\section{Introduction}

Nasopharyngeal carcinoma (NPC) is a leading lethal malignancy with a high prevalence in Southeast Asia, especially in the Guandong and Guangxi provinces in Southern China [1-2]. Most NPC tumor cells are poorly differentiated or undifferentiated and have a high tendency to invade adjacent regions and me- tastasize to neck lymph nodes. Although early-stage NPC is radio-curable, NPC patients at late-stage have poor outcomes, largely due to local failure in treatments and distant metastases [3]. Conventional TNM staging has strong prognostic significance for NPC [4], yet the prognosis remains poor in a significant 
number of NPC patients at late-stage [5]. Therefore, it's necessary to identify additional marker(s) for predicting the clinical prognosis as well as serving as optimal therapeutic target(s) to benefit NPC patients.

Human leukocyte antigen G (HLA-G) is a non-classical major histocompatibility complex (MHC) class Ib antigen. In non-pathological situations, HLA-G expression is restricted to the fetal-maternal interface of the extravillous cytotrophoblasts, placental chorionic endothelium, thymic epithelial cells, and erythropoietic lineage cells from the bone marrow [6], as well as other immune-privileged tissues such as the cornea [7], nail matrix [8], and autologous tissues such as the pancreas [9]. It has been demonstrated that HLA-G plays important role in immune tolerance during pregnancy. It can suppress the proliferation of alloreactive $\mathrm{CD} 4^{+} \mathrm{T}$-cell [10-12], block the effector function of decidual monocyte/macrophages [13], inhibit cytolysis mediated by NK-cell and T cell [11, 14], modulate the release of cytokines and shift decidual mononuclear cells toward the T helper (Th) 2 profile. Of the Th2 cytokines, interleukin (IL)-10 has been shown to induce HLA-G expression, which in return stimulates IL-10 expression [15-17]. Interestingly, HLA-G expression was detected in various types of human malignancies, and has been correlated with certain clinicopathological parameters in gastric carcinoma [18], lymphoma [19-20], ovarian [21] and endometrial carcinoma [22]. Moreover, recent studies have suggested that HLA-G may promote tumor progression by suppressing immune regulation within tumor microenvironment, and thus helping tumor cells escape from anti-tumor immune surveillance [23]. Therefore, these studies suggest that HLA-G might serve as a clinical marker for the prediction of clinical outcomes of these diseases [24-26].

Most recently, the polymorphisms of HLA-G were associated with NPC risk and clinical outcome [27]. The Ile110 allele was found to be significantly less frequent among patients with a positive lymph node status and late stages (III-IV), respectively [27]; moreover, the occurrence of $130 \mathrm{C}$ deletion was significantly associated with a decreased NPC free disease and overall survival [27]. However, to date, the expression and role of HLA-G in NPC have not been investigated.

Here, in order to explore the role of HLA-G in NPC, we first examined HLA-G expression in NPC using immunohistochemistry (IHC) and immunoblot (western) methods; and secondly, we analyzed the correlation between the HLA-G expression level and the clinical factors and outcomes of NPC patients; and finally, we evaluated the possible immune-regulatory function of HLA-G in the tumor microenvironment in NPC.

\section{Methods and Materials}

\section{Patients and clinical tissue samples}

In this study, 552 specimens of NPC were collected at Sun Yat-Sen University Cancer Center, Guangzhou, China between January 2001 and December 2003. The cases were selected based on the following criteria: pathologically confirmed NPC with available biopsy specimens for tissues microarray (TMA) construction; no previous malignant disease or a second primary tumor; no prior radiotherapy, chemotherapy or surgery treatment history. All of the selected NPC samples contained at least $70 \%$ carcinoma tissue, as determined by examining of the frozen section. The characteristics of the NPC patients were summarized in Table 1.

Table I. Characteristics of nasopharyngeal carcinoma patients

\begin{tabular}{ll}
\hline Characteristic & $\begin{array}{l}\text { Nasopharyngeal carcinoma patients } \\
\text { (N=552)\% }\end{array}$ \\
\hline Sex & $134(24.3)$ \\
Female & $418(75.7)$ \\
Male & \\
Age (years) & $46(19-78)$ \\
Median(range) & $46.78 \pm 11.065$ \\
Mean+SD & \\
Clinical stage & $147(26.6)$ \\
I - II & $405(73.4)$ \\
III-IV & \\
Progression & $335(60.7)$ \\
No & $217(39.3)$ \\
Yes & \\
Death & $368(66.7)$ \\
No & $184(33.3)$ \\
Yes & \\
Follow-up time (months) & $67(2-99)$ \\
Median(range) & $58.63 \pm 1.097$ \\
Mean+SD & \\
WHO histological classifi- & \\
cation & $400(72.5)$ \\
NKUC & $139(25.2)$ \\
NKDC & $13(0.3)$ \\
KSCC & \\
\hline Note: & \\
\hline
\end{tabular}

Note: SD, standard deviation; WHO, World Health Organization; NKUC, non-keratinizing undifferentiated carcinoma; NKDC, non-keratinizing differentiated carcinoma; KSCC, keratinizing squamous cell carcinoma. 
All patients were treated with standard curative radiotherapy, with or without chemotherapy. The follow-up period was defined as the time from diagnosis to the date of death or the lasted census date of living patients. Disease progression was defined as cases in which the tumor was evaluated as progressive disease after primary treatment or recurrence (local progression) and/or cases in which new distant metastasis occurred (distant progression). The Institute Research Medical Ethics Committee of Sun Yat-Sen University granted approval for this study.

\section{Tissue microarray construction}

The paraffin-embedded specimens were obtained from the previously constructed tissue microarray (TMA) [28]. Briefly, the paraffin-embedded tissue blocks and the corresponding hematoxylin and eosin (H\&E) stained slides were overlaid for TMA sampling. Duplicates of $1.0 \mathrm{~mm}$ diameter cylinders were punched from representative tumor areas of individual tissue block and re-embedded into a recipient paraffin block at a defined position using a tissue-arraying instrument (Beecher Instruments, Silver Spring, MD).

\section{Immunohistochemistry (IHC) and evaluation}

IHC was performed to examine HLA-G, CD68 and IL-10 expression in NPC tissue specimens. Primary antibodies against HLA-G (1:200 dilution, sc-21799, Santa Cruz, USA), CD68 (1:100 dilution, ABIN370601, Dako, Denmark) and IL-10 (1:300 dilution, ab34843, Abcam, USA) were used in this study, following the previous protocol [28]. The IHC results were evaluated and scored independently by three pathologists without prior knowledge of the clinicopathological outcomes of the patients. A semi-quantitative estimation was made using a composite score obtained by adding the values of the staining intensity and the relative abundance of the positive cells. The intensity was graded as 0 (negative), 1 (weakly positive), 2 (moderately positive) or 3 (strongly positive). The abundance of the positive cells was graded from 0 to 4 according to their percentages $(0,<5 \% ; 1,5-25 \% ; 2,26-50 \% ; 3,51-75 \% ; 4$, $76-100 \%)$. The concordance rate of $83.4 \%$ among the evaluated results from the pathologists demonstrated that this scoring method was highly reproducible. The value was selected until at least two pathologists reported consistent results. In cases where scoring was completely different, all of the pathologists worked together to confirm the score. In addition, the numbers of $\mathrm{CD}^{2} 8^{+}$macrophages in tumor nest and stroma were counted within the areas of 6 high-power fields (400X).

\section{Selection of cutoff score}

Receiver operating characteristic (ROC) curve analysis was performed to determine the cutoff score for a "high expression" designation with the 0,1-criterion implemented in SPSS software [28]. First, the clinicopathologic characteristics were dichotomized as following groups: $\mathrm{T}$ classification (T1-T2 versus T3-T4), N classification (N0 versus N1-N3), clinical stage (I-II versus III-IV), cancer progression (Yes versus No), and survival status (death due to NPC versus censored). Second, the expression scores for HLA-G were trained in the ROC analysis. The cutoff score is the point on the curve that has both maximum sensitivity and specificity [28-29].

\section{Cell lines and cell culture}

Eight NPC cell lines including CNE2, S26, S18, SUNE1, 6-10B, 5-8F, CNE1 and HONE1 were cultured in RPMI 1640 (Gibco, USA) with 10\% fetal bovine serum (Gibco, USA). The NPC cell line C666 was cultured in RPMI 1640 medium (Gibco, USA) containing $15 \%$ FCS. The immortalized nasopharyngeal epithelial cell line NP69 was cultured in keratinocyte serum-free medium (Invitrogen) supplemented with 5\% FCS, $25 \mu \mathrm{g} / \mathrm{ml}$ bovine pituitary extract, and $0.2 \mathrm{ng} / \mathrm{ml}$ recombinant epidermal growth factor, as suggested by the manufacturer. All the cell lines were grown in a humidified incubator at $37^{\circ} \mathrm{C}$ with $5 \% \mathrm{CO}_{2}$.

\section{Immunoblot analysis}

Cells were harvested and lysed with RIPA buffer (Upstate, USA). Equal amount of denatured protein samples were separated by SDS-PAGE and then transferred to PVDF membranes (Amersham, UK) for immunoblot or western analysis. Antibodies used for immunoblot analysis were against HLA-G (same as the one used in IHC), and anti-a-tubulin antibody (1:1,000 dilution, G436, Bioworld, USA) was used as loading control. All protein bands were visualized using an enhanced chemiluminescent western blot Kit (Amersham, UK).

\section{Statistical analysis}

Statistical analysis was performed using SPSS (version 16.0; SPSS, Chicago, IL). ROC curve analysis was applied to determine the cutoff score for high expression of HLA-G. The correlation between HLA-G expression and clinicopathologic features of NPC patients was analyzed by $\chi^{2}$-test. For univariate survival analysis, survival curves were obtained using the Kaplan-Meier method. In multivariate statistical analysis, we treated overall survival time as "time", overall survival status as "status", "death" as the reference (with a value of 1), while the expression of 
HLA-G and other significant clinicopathologic features (age, $\mathrm{T}$ classification, $\mathrm{N}$ classification, recurrence or metastasis and clinical stage) were treated as the comparing parameters (with a relative value), respectively. The Cox proportional hazards regression model was used to identify independent prognostic factors. Associations between the HLA-G expression and IL-10 expression or number of $\mathrm{CD}^{+} 8^{+}$macrophages were examined by using Pearson correlation and independent t-test. Differences were considered significant if the $P$-value from a two-tailed test was less than 0.05 .

\section{Results}

\section{Patient characteristics}

Among the 552 patients, 418 (75.7\%) were male, and the median age was 46 years (ranging 19-78 years). Among these patients, 139 patients (25.2\%) were diagnosed as non-keratinizing differentiated carcinoma (NKDC), 400 patients $(72.5 \%)$ were diagnosed with non-keratinizing undifferentiated carcinoma (NKUC), and a few patients $(0.3 \%)$ were diagnosed with keratinizing squamous cell carcinoma
(KSCC). One hundred forty-seven patients $(26.6 \%)$ were in stage I and stage II, and 405 patients (73.4 \%) were in stage III and stage IV. The median follow-up time was 58.63 months (ranging 2 to 99 months). Of the total number of patients, 184 (33.3\%) died and 217 $(39.3 \%)$ presented with disease progression during the five-year follow-up period. Detailed clinical information is shown in Table 1.

\section{Expression of HLA-G in NPC cell lines and tumor tissues}

To test the specificity of HLA-G antibody for the detection of HLA-G expression in NPC, western-blot assays were performed to evaluate the protein levels of HLA-G in the NPC cell lines including CNE2, S26, S18, SUNE1, 6-10B, 5-8F, CNE1, HONE1, C666 and an immortalized primary nasopharyngeal epithelial cell line NP69. Compared to non-tumorous NP69 cells, NPC cell-lines expressed higher levels of HLA-G (Figure. 1A). Moreover, a single targeted band with clear background for each NPC sample indicates high specificity of the antibody against HLA-G, in consistent with manufacturer's statement.
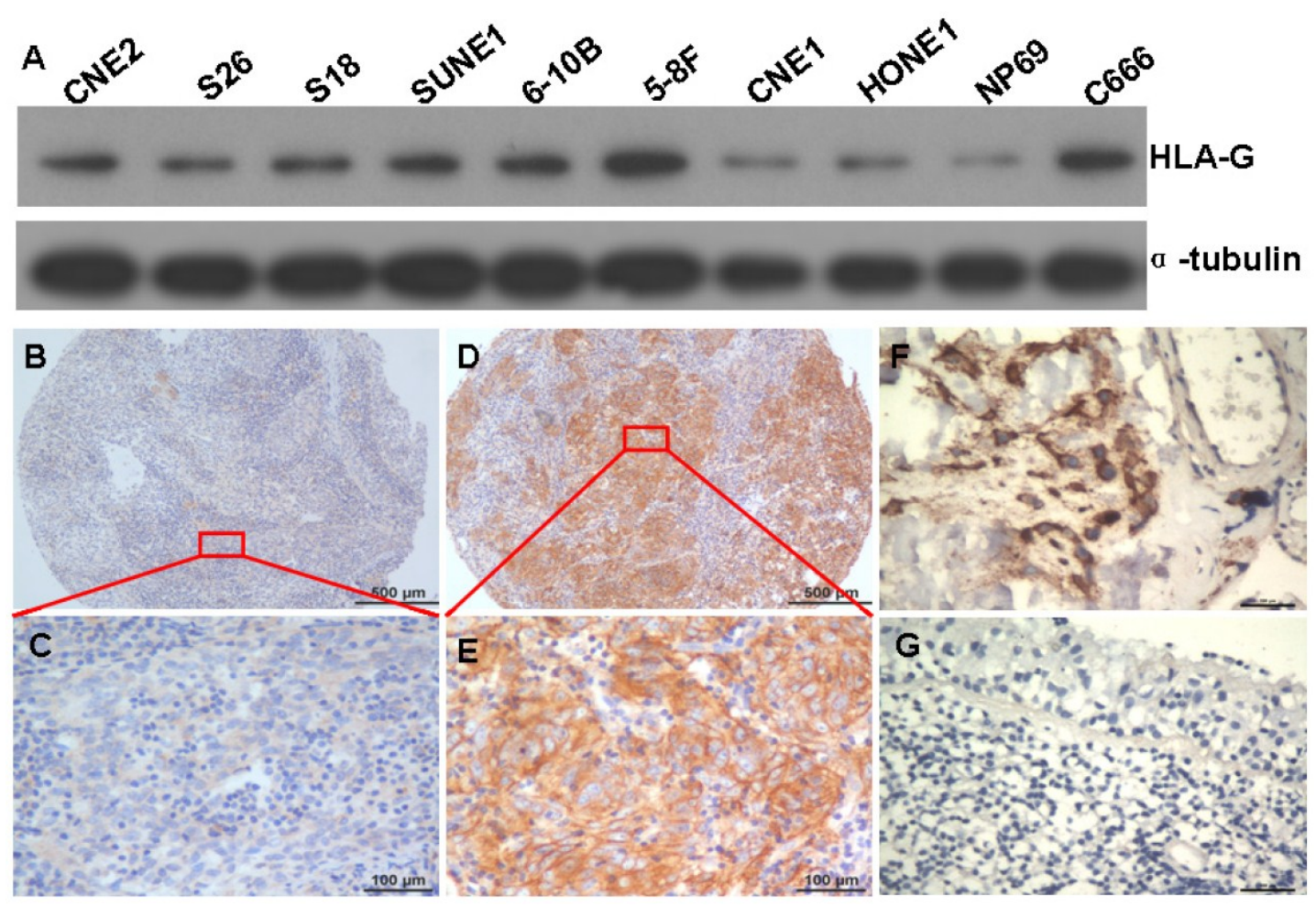

Figure I. Expressions of HLA-G in NPC cell lines and NPC tissues. A The HLA-G protein was visualized by western blot in the NPC cell lines CNE2, S26, SI8, SUNEI, 5-8F, 6-IOB, CNEI, HONEI, NP69 and C666 cells (molecular weight, HLA-G: 39KD, $\alpha$-tubulin: 50 KD). B and $C$ Weak HLA-G staining in TMA samples under low and high magnifications; D and E Strong HLA-G staining in TMA samples are shown under low and high magnifications; $F$, strong HLA-G staining in placenta tissue (positive control); G No HLA-G staining in normal nasopharyngeal epithelium tissue (negative control). 

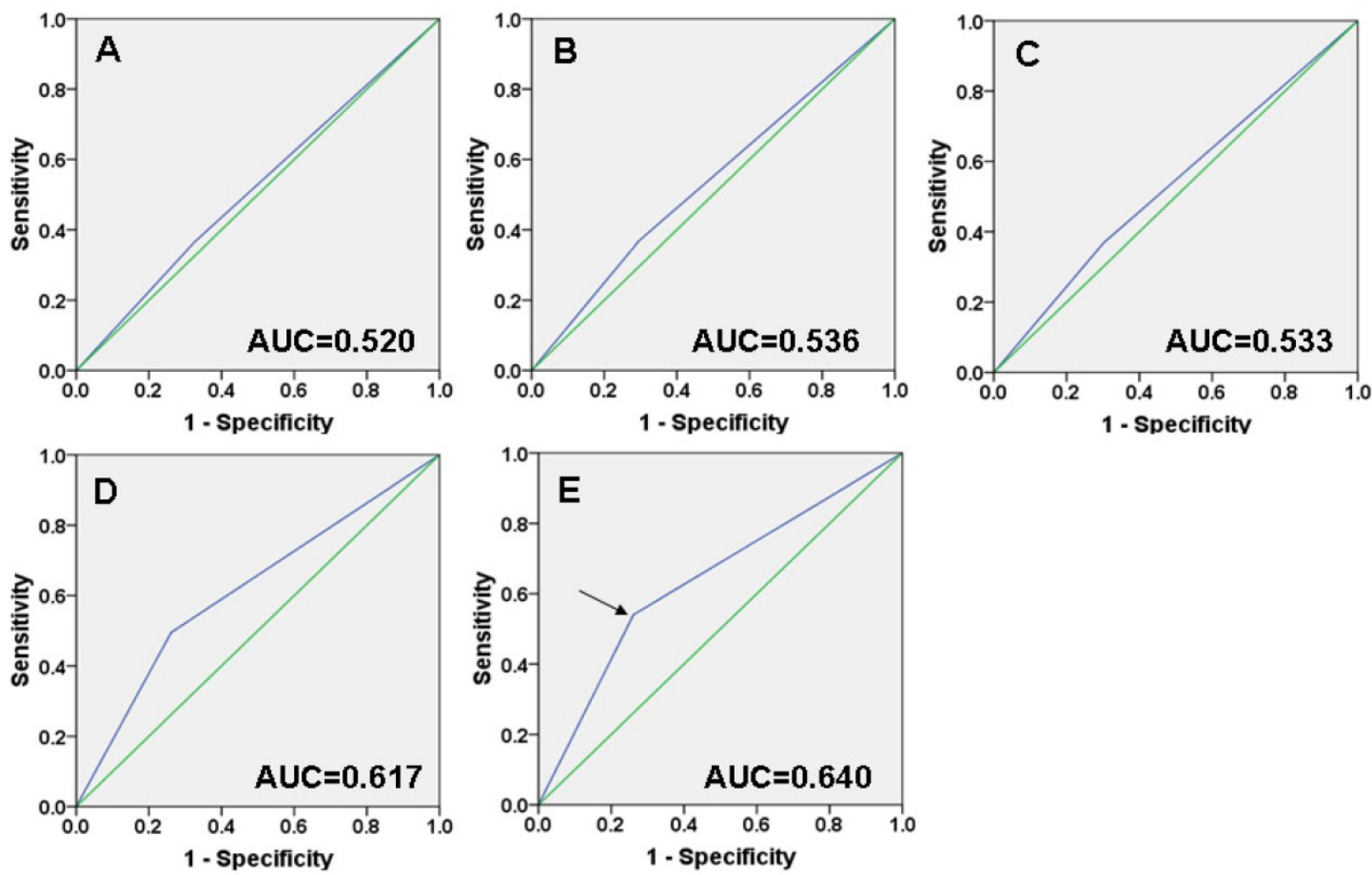

Figure 2. Selection of the cutoff score. Receiver operating characteristic curve analysis was employed to determine the cutoff score for the designation of "high expression" of HLA-G. The sensitivity and specificity for each outcome of HLA-G membranal and cytoplasmic staining were plotted: A T classification $(P=0.431)$; B N classification $(P=0.191)$; C Clinical stage $(P=0.23 \mathrm{I})$; $\mathrm{D}$ Cancer progression $(P$ $<0.00 \mathrm{I})$; E Survival status $(P<0.00 \mathrm{I})$.

Subsequently, the expression of HLA-G protein was determined in 552 NPC tumors from which five-year follow-up information were available, by using IHC with the specific antibody. HLA-G was detected in 437 of 552 (79.2\%) NPC cases. Overexpression of HLA-G was observed in the membrane and cytoplasm of NPC cells with different intensity (Figure $1 \mathrm{~B}, \mathrm{C}, \mathrm{D}$ and $\mathrm{E}$ ) and strong staining in placenta tissue (positive control) (Figure 1F). However, no positive signal was observed in normal nasopharyngeal epithelium with this antibody (Figure 1G), indicating again the specificity of the antibody and more importantly, lack of HLA-G expression in normal tissue. Immunoreactivity for HLA-G was scored for subsequent evaluation of its predictive value in NPC.

\section{Selection of the cutoff score for high expres- sion of HLA-G}

ROC curve analysis showed that HLA-G expression has some sort of predictive value in NPC, with maximum area under curve (AUC) reaching 0.640 (Figure. 2E, black arrowed). The points in curves with both maximum specificity and maximum sensitivity were treated as cutoff points for high or low expression of HLA-G. For survival analysis the cutoff score for the expression of HLA-G was 4.5 (Table 2). Thus, tumors designated as low expression of HLA-G are those with the scores below or equal to the cutoff score, while tumors of high expression are those with scores above the cutoff score.

Table 2. The corresponding cutoff score of HLA-G expression for each clinicopathological feature according to ROC curve analysis

\begin{tabular}{lll}
\hline Feature & Cutoff score & P value \\
\hline T classification & 1.5 & 0.431 \\
N classification & 5.5 & 0.191 \\
Clinical stage & 1.5 & 0.231 \\
Cancer progression & 4.5 & $<0.001$ \\
Survival status & 4.5 & $<0.001$ \\
\hline
\end{tabular}

Note: HLA-G, human leukocyte antigen G; ROC, receiver operating characteristic.

\section{Association of HLA-G expression with clini- copathologic features and survival}

As mentioned above and previously [28], samples were separated into either low or high HLA-G expression groups, according to their expression levels to the cutoff. As a result, high expression of HLA-G was observed in 187/552 (33.9\%) of NPC tissues. With $\chi^{2}$-test analysis, we found that high expression of HLA-G was positively correlated with 
tumor $\mathrm{N}$ classification (Pearson Correlation $=0.120$; $P=0.005$ ), recurrence or metastasis (Pearson Correlation $=0.229 ; P<0.001)$ and histological type (Pearson Correlation $=0.166 ; P<0.001$ ), respectively (Table 3 ); while, there was no significant association between HLA-G expression and other clinicopathologic features, such as patient gender, age, tumor $\mathrm{T}$ classification and clinical stage $(P>0.05$, Table 3$)$.

Table 3. Association of HLA-G expression and clinicopathological characteristics in NPC patients

\begin{tabular}{|c|c|c|c|}
\hline \multirow[t]{2}{*}{ Characteristics } & \multicolumn{2}{|c|}{ HLA-G IHC staining } & \multirow[t]{2}{*}{$P$ value } \\
\hline & $\begin{array}{l}\text { low expression } \\
(\mathrm{N}=365)\end{array}$ & $\begin{array}{l}\text { high expression } \\
(\mathrm{N}=187)\end{array}$ & \\
\hline \multicolumn{3}{|l|}{ Age, years } & 0.302 \\
\hline$<46$ & 177 & 82 & \\
\hline$>=46$ & 188 & 105 & \\
\hline \multicolumn{3}{|l|}{ Gender } & 0.071 \\
\hline Male & 285 & 133 & \\
\hline Female & 80 & 54 & \\
\hline \multicolumn{3}{|l|}{ Tumor stage } & 0.974 \\
\hline $\mathrm{T} 1+\mathrm{T} 2$ & 143 & 73 & \\
\hline $\mathrm{T} 3+\mathrm{T} 4$ & 232 & 54 & \\
\hline \multicolumn{3}{|c|}{ Lymphoid Nodal(N)states } & 0.005 \\
\hline N0 & 100 & 44 & \\
\hline N1-3 & 265 & 143 & \\
\hline \multicolumn{3}{|c|}{ Recurrence or metastasis } & $<0.001$ \\
\hline Yes & 127 & 90 & \\
\hline No & 238 & 97 & \\
\hline \multicolumn{3}{|c|}{ TNM clinical stage } & 0.807 \\
\hline $\mathrm{I}+\mathrm{II}$ & 96 & 51 & \\
\hline $\mathrm{III}+\mathrm{IV}$ & 269 & 136 & \\
\hline \multicolumn{3}{|c|}{ Histological type } & $<0.001$ \\
\hline NKUC & 283 & 114 & \\
\hline NKDC & 82 & 73 & \\
\hline
\end{tabular}

Note: HLA-G, human leukocyte antigen G; ROC, receiver operating characteristic.

The 5-year overall and disease-free survival rate of the cohort of 552 NPC patients were $66.6 \%$ and $60.6 \%$, respectively (Figure 3A and 3B). Of these 552 NPC patients, Kaplan-Meier and log-rank test analyses indicated that high HLA-G expression significantly associated with poor overall survival (OS) (5-year survival rates, $57.2 \%$ vs. $70.1 \%$, log-rank test, $\mathrm{X}^{2}=10.324, \mathrm{P}=0.001$, Figure $3 \mathrm{C}$ ) and disease-free survival (DFS) (5-year survival rates, $51.9 \%$ vs. $64.8 \%$, log-rank test, $\mathrm{X}^{2}=8.604, \mathrm{P}=0.003$, Figure 3D) compared with low HLA-G expression. When the clinical stages were taken as stratifications, the associations are much significantly stronger for patients at late stages (stage III - IV), between HLA-G expression and poor OS (5-year survival rates, $42.6 \%$ vs. $64.1 \%$, log-rank test, $\mathrm{X}^{2}=17.563, \mathrm{P}<0.001$, Figure $3 \mathrm{G}$ ) and DFS (5-year survival rates, $39.0 \%$ vs. $59.0 \%$, log-rank test, $\mathrm{X}^{2}=14.684, \mathrm{P}<0.001$, Figure $3 \mathrm{H}$ ); while the associations are not significant for patients at the early stages (stage I - II; Figure 3E and 3F).

\section{Independency test of the association between HLA-G expression and survival}

A univariate Cox proportional hazard regression analysis showed that HLA-G expression $(\mathrm{P}<0.001)$, age $(\mathrm{P}=0.002)$ and the well-known clinicopathologic prognostic parameters, such as $\mathrm{T}$ classification $(\mathrm{P}<0.001), \mathrm{N}$ classification $(\mathrm{P}=0.001)$, recurrence or metastasis $(\mathrm{P}<0.001)$ and clinical stage $(\mathrm{P}<0.001)$, were significantly associated with survival (Table 4). Moreover, independency tests were carried out among the prognostic factors, including the expression of HLA-G and other clinicopathologic features (age, $\mathrm{T}$ classification, $\mathrm{N}$ classification, recurrence or metastasis and clinical stage), under a multivariate Cox proportional hazards regression model (Table 4). Compared with above significant clinicopathologic features (age, $\mathrm{T}$ classification, $\mathrm{N}$ classification, recurrence or metastasis and clinical stage), HLA-G was an independent and unfavorable factor for overall patient survival (hazards ratio: 1.429; 95\% confidence interval: 1.050-1.945; $\mathrm{P}=0.023$ ). Besides, age, recurrence or metastasis and clinical stage, respectively, were found to be independent prognostic predictors for overall survival (Table 4).

\section{Correlations of HLA-G expression and CD68+ macrophage and IL- 10 in NPC tissue}

To elucidate the roles of HLA-G in immune regulation, we analyzed the correlation between expression of HLA-G and the number of $\mathrm{CD}^{+} 8^{+}$macrophage in tumor nest and stroma, which has been implicated in tumor microenvironment [30]. Among 552 NPC tissues, HLA-G high expressions were inversely correlated with infiltrations of $\mathrm{CD}^{+} 8^{+}$macrophages in tumor nest (19.53 \pm 11.09/HPF vs, $21.89 \pm 12.86 / \mathrm{HPF}$ Pearson Correlation $=-0.086 ; P=0.025$ ) and in tumor stroma $(43.73 \pm 22.11 / \mathrm{HPF}$ vs, $50.66 \pm 20.93 / \mathrm{HPF}$ Pearson Correlation=-0.107; $P<0.001$; Figure 4), respectively, which is in consistent with previous report [13]. Moreover, the correlation between the expression of HLA-G and IL-10 in the NPC samples were also examined, inspired by the previous demonstrations that IL-10 as an important cytokine can induce HLA-G expression in other tumors [31]. The results showed a positive correlation between the expression of HLA-G and IL-10 in 110 NPC tissues (Pearson 
Correlation $=0.743 ; P<0.001$ ) (Figure 5A). For the 52 NPC cases with a high expression of HLA-G, the IL-10 average staining score was 5.67 , which was signifi- cantly higher than that found in the remaining 58 NPC samples with low expression of HLA-G (3.90) $(P<0.001$, independent sample t-test; Figure 5B).

Table 4 Univariate and multivariate Cox regression analyses of different prognostic variables in patients with NPC

\begin{tabular}{llll}
\hline Variable & Subset & Hazard ration (95\%) CI & P value \\
\hline Univariate analysis $(\mathbf{N}=\mathbf{5 5 2})$ & & & $<.629(1.217-2.180)$ \\
HLA-G IHC staining & low vs. high & $1.613(1.198-2.172)$ & 0.001 \\
Age & $<46$ vs. >=46 & $0.835(0.587-1.190)$ & 0.002 \\
Gender & male vs. female & $3.32(2.296-4.801)$ & $<0.001$ \\
T stage & T1+T2 vs. T3+T4 & $2.077(1.404-3.071)$ & 0.001 \\
N stage & N0 vs. N1+N2+N3 & $606.868(84.874-4.339 \mathrm{E} 3)$ & $<0.001$ \\
Recurrence or metastasis & No vs. Yes & $1.179(0.894-1.554)$ & 0.243 \\
Histological type & NKUC vs. NKDC & $7.271(3.951-13.380)$ & $<0.001$ \\
Clinical stage & I + II vs. III+IV & & 0.023 \\
Multivariate analysis $(\mathbf{N}=552)$ & & $1.429(1.050-1.945)$ & 0.011 \\
HLA-G IHC staining & low vs. high & $1.496(1.098-2.039)$ & 0.939 \\
Age & $<46$ vs. >=46 & $0.982(0.622-1.551)$ & 0.305 \\
T stage & T1+T2 vs. T3+T4 & $1.240(0.822-21.871)$ & $<0.001$ \\
N stage & N0 vs. N1+N2+N3 & $494.208(68.913-3.54 \mathrm{E} 3)$ & 0.016 \\
Recurrence or metastasis & No vs. Yes & $2.471(1.184-5.159)$ & \\
Clinical stage & I + II vs. III+IV & & \\
\hline
\end{tabular}

Note: CI, confidence interval; WHO, World Health Organization; HLA-G, human leukocyte antigen G.
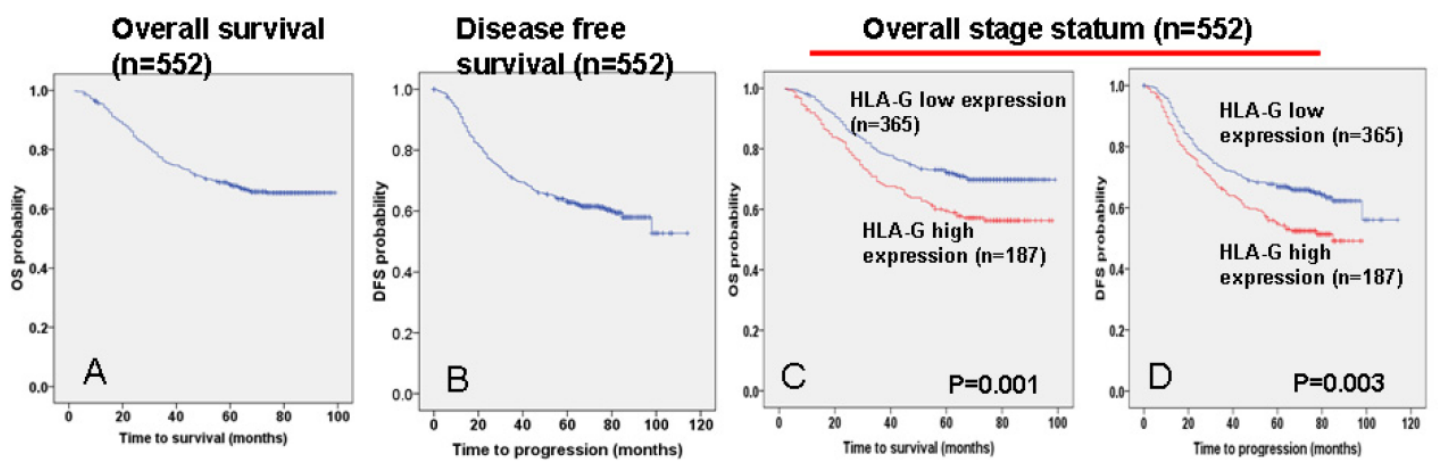

Early stage statum ( $\mathrm{n}=147)$
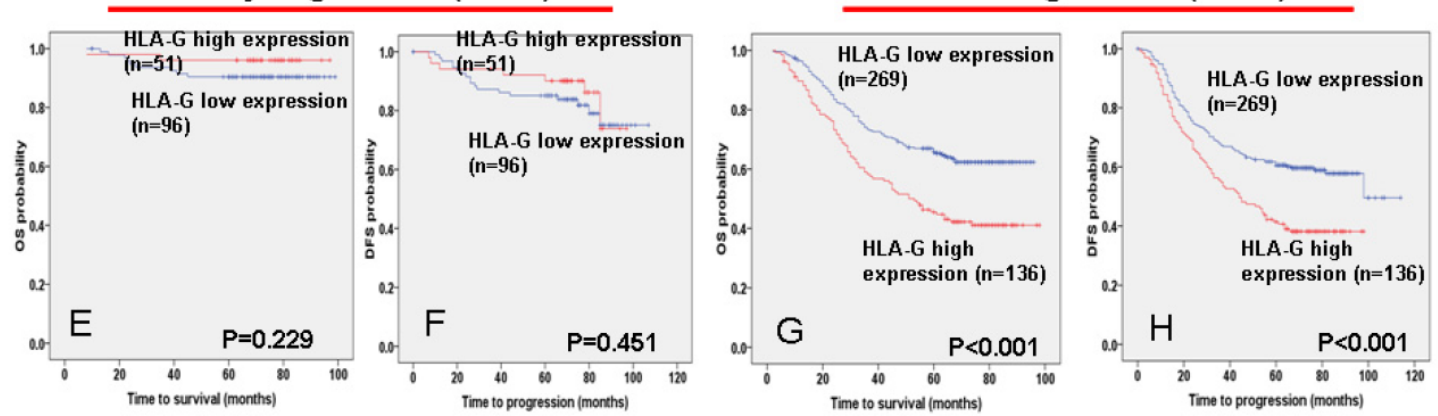

Figure 3. Association between HLA-G expression and NPC patient survival. TMA analyses were conducted in a cohort of 552 NPC patients diagnosed at M0. A The five-year overall survival (OS) rate of was $66.6 \%$. B The five-year disease-free survival (DFS) rate of was 60.6\%. C and D High HLA-G expression levels were significantly associated with OS $(P=0.00 \mathrm{I})$ and disease-free survival $(P=0.00 \mathrm{I})$ in all NPC patients. E and F No significant differences in five-year OS and DFS rates were found between groups with low and high expressions of HLA-G in NPC patients at early stages $(\mathrm{I}-\mathrm{II})$. G and H High HLA-G expression levels were significantly associated with OS $(\mathrm{P}=0.006)$ and disease-free survival $(P=0.007)$ in NPC patients at late stages $(I I I-I V)$. 

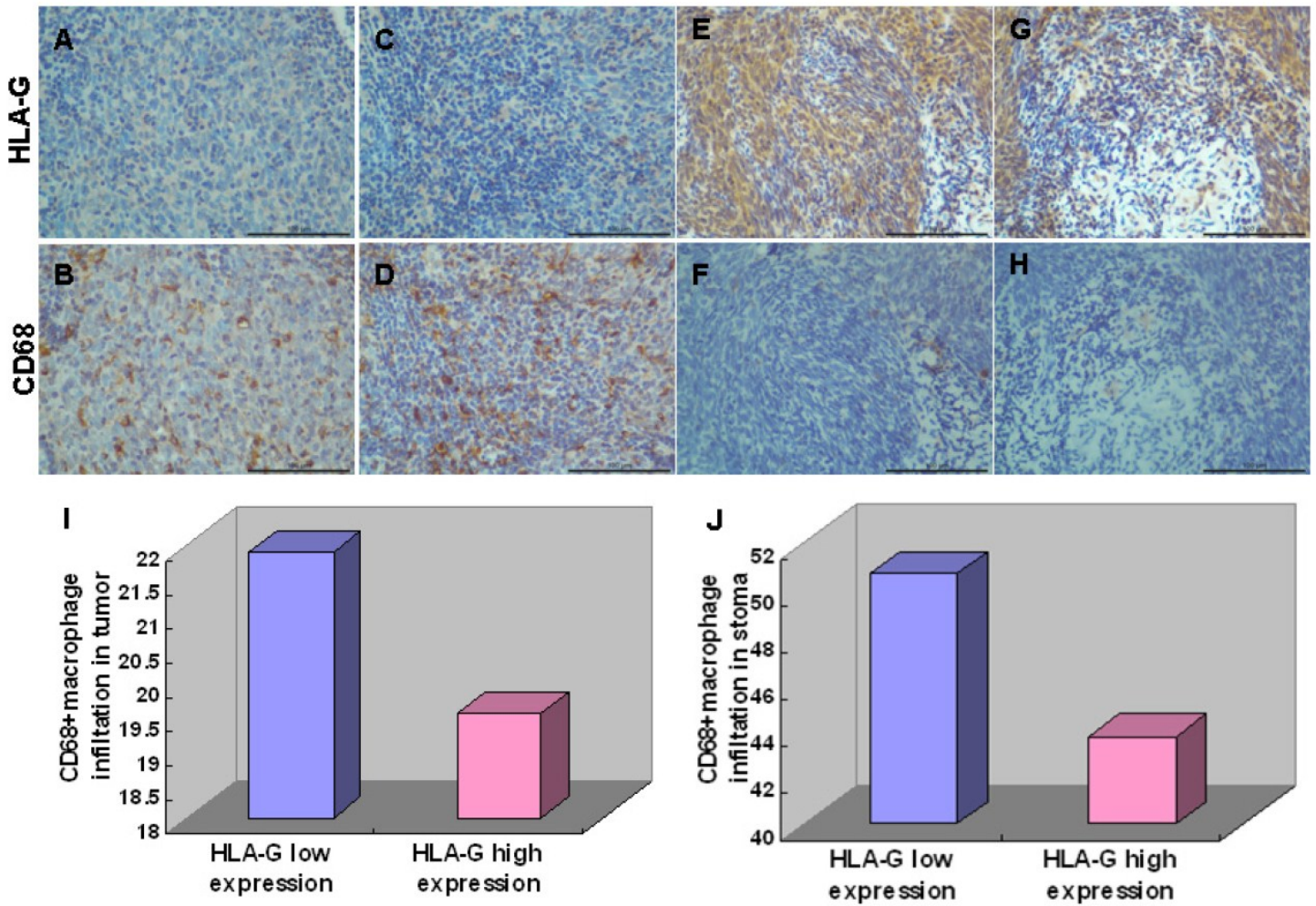

Figure 4. Immunohistochemical staining of HLA-G expression and CD68+ macrophage. Representative pictures were shown. Weak HLA-G staining in tumor nest $(A)$ and stroma $(C)$ correlated with increasing infiltrations of CD68+ macrophages in tumor nest (B) and stroma (D); Strong HLA-G staining in tumor nest $(E)$ and stroma $(G)$ correlated with increasing infiltrations of $C D 68^{+}$macrophages in tumor nest $(F)$ and stroma $(H)$; High expression of HLA-G significantly decreased infiltrations of $\mathrm{CD}^{2} 8^{+}$macrophages in tumor nest (I) and tumor stroma $(J)$ compared with low expression of HLA-G.
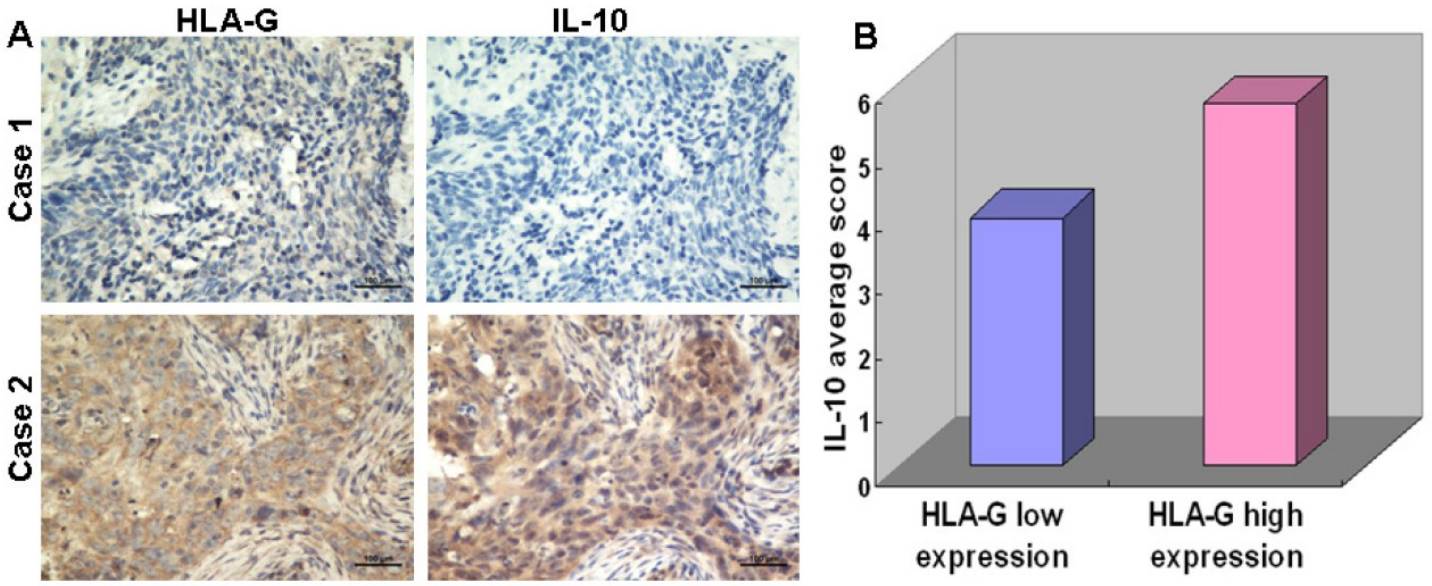

Figure 5. IHC analysis showing correlation between the expression levels of HLA-G and IL-I0 in NPC tissues. A Continuous sections of human NPC tissue were subjected to IHC staining with antibodies against HLA-G and IL-I0. The tumor tissue in case I showed low expressions of HLA-G and IL-10; while the tumor tissue of case 2 showed the high expression levels of HLA-G and an elevated IL-10. B In 52 NPC cases with high expression levels of HLA-G, the average HLA-G score was 5.67 (right column), which was significantly higher than that (3.90) of the 58 NPCs with low expression levels of HLA-G (left column, $P<0.00$ I, independent sample $t$-test). 


\section{Discussion}

NPC is a malignancy arising at the upper lining of nasopharynx, and the strategies for early diagnosis and prognosis are keys for NPC survival. As nasopharynx is an important lymphoid tissue [32], immune-related genes might contribute to the development of NPC. Indeed, some immune-related genes especially the human leukocyte antigen (HLA) genes have been implicated in the NPC susceptibility [33]. It has recently been reported that HLA-G polymorphisms are associated with NPC risk and clinical outcome [27]. However, to date, the expression and role of HLA-G in NPC have not been investigated.

Here, we found that overexpression of HLA-G protein is likely correlated with NPC. The expression of HLA-G protein was higher in all the NPC cell lines tested, but low in the immortalized nasopharyngeal epithelial cell line NP69; and moreover, HLA-G protein was expressed in $79.2 \%$ of 552 NPC patient specimens, but not in normal nasopharyngeal epithelium tissue (Figure 1). These suggested that HLA-G might play an important role in the tumorigenesis of NPC. Supportively, the overexpression of HLA-G antigens has been detected in various types of human malignancies, including gastric carcinoma [18], lymphoma [19-20], ovarian [21] and endometrial carcinoma [22].

The expression of HLA-G might contribute to the progression of NPC. Among the NPC patients, high expression of HLA-G was correlated with tumor N classification, recurrence and metastasis and histological type, respectively $(\mathrm{P}<0.05$, Table 3$)$, which are well-known factors related to NPC progression as well as survival. Moreover, high expression of HLA-G was correlated with poor disease free and overall survival rates of NPC. For patients at late stages, the strength of associations between high HLA-G expression and poor survivals is much enhanced. These suggest that HLA-G might participate in the regulation of NPC progression, and with more contribution in the advanced tumors. Importantly, the correlation of HLA-G expression with NPC survival and the identification of HLA-G as an independent and unfavorable prognostic factor of NPC indicate that HLA-G could be a useful biomarker for NPC prognosis. Further replication study using larger sample size might be necessary, before it's applied to clinical usage.

Considering the immunomodulatory function of HLA-G, its overexpression in malignant cells may represent one of various mechanisms adopted by the tumor cells to escape immune surveillance [31, 34]. Studies have shown that HLA-G expression can pro- tect tumor cells from cytolysis mediated via natural killer cell (NK) and cytotoxic T lymphocyte (CTL) [35] and block the effector function of decidual monocyte/macrophages [13]. To better understand the possible role of HLA-G involved in NPC, we further determine its correlations with $\mathrm{CD}^{+} 8^{+}$macrophage and IL-10, which are also prognostic immune molecules in NPC and play important role in the immune surveillance [30], [36-37]. We found a significant reverse correlation between HLA-G expression and infiltrations of $\mathrm{CD} 68^{+}$macrophage in NPC tumor nest and tumor stroma tissues (Pearson Correlation $=-0.086$ and -0.107 , respectively). It has been shown that $\mathrm{CD}^{+} 8^{+}$macrophage was able to secret tumor necrosis factor-alpha and killed tumor cells, and the anti-tumor ability was promoted when it was co-cultured with supernatant of NPC cells [30]. These results suggest a link between HLA-G and tumor associated macrophages regarding their regulatory roles in immune surveillance in NPC.

On the other hand, we found that IL-10 was highly expressed in NPC tissues, consistent with previous reports [36-37]. Moreover, the expression of IL-10 was positively correlated with that of HLA-G in NPC tissues. Similarly, the expression of IL-10 has been demonstrated in 7 of 9 HLA-G positive cases with lung cancer [38]. These suggest a close functional relevance between IL-10 and HLA-G. As a supportive evidence, it has been found that IL-10 could induce HLA-G in human trophoblasts and mononuclear cells [15]; and in turn, HLA-G has cytokine-regulating functions, which is associated with IL-10 expression in the evasion of immune surveillance [31]. Since IL-10 has anti-inflammatory and immunosuppressive activities, HLA-G might contribute to these processes, and interact with IL-10 during the malignant transformation of NPC.

In summary, we have demonstrated that HLA-G is expressed in majority of NPC samples; and that HLA-G expression is a strong and independent prognostic factor in NPC. Moreover, the correlations of HLA-G with other two prognostic factors, CD68 ${ }^{+}$ macrophage and IL-10, were revealed in NPC tissues, suggesting that HLA-G might regulate the escape of tumor surveillance in the NPC. However, further investigations are necessary to reveal the precise function of HLA-G in NPC development.

\section{Acknowledgements}

This study was supported partially by the National High Technology Research and Development Program of China (863 Program) (No. 2012AA02A206 and 2012AA02A501), and the State Basic Research Development Program of China (973 Program): (No. 


\section{CB504300 and 2011CB504805).}

\section{Authors' Contributions}

MB conducted all the experiments, and wrote the initial draft and revised the manuscript. HQ collected the preliminary data, and helped to perform some experiments. JJ and QC counted the number of macrophage in NPC tissues. CC, TB and JY participated in the study design and interpretation of the results. QS, HY, YL and LZ undertaking immunohistochemistry work. JX and YX contributed in the study coordination and revisions. All authors read and approved the final manuscript.

\section{Competing Interests}

The authors declare no competing interest.

\section{References}

1. Wei WI, Sham JS. Nasopharyngeal carcinoma. Lancet. 2005; 365: 2041-54.

2. Chang ET, Adami HO. The enigmatic epidemiology of nasopharyngeal carcinoma. Cancer Epidemiol Biomarkers Prev. 2006; 15: 1765-77.

3. Chua DT, Ma J, Sham JS, Mai HQ, Choy DT, Hong MH, et al. Long-term survival after cisplatin-based induction chemotherapy and radiotherapy for nasopharyngeal carcinoma: a pooled data analysis of two phase III trials. J Clin Oncol. 2005; 23: 1118-24.

4. Patel SG, Shah JP. TNM staging of cancers of the head and neck: striving for uniformity among diversity. CA Cancer J Clin. 2005; 55: 242-58.

5. Ma BB, Chan AT. Recent perspectives in the role of chemotherapy in the management of advanced nasopharyngeal carcinoma. Cancer. 2005; 103: 22-31.

6. Carosella ED, Moreau P, Le Maoult J, Le Discorde M, Dausset J, Rouas-Freiss N. HLA-G molecules: from maternal-fetal tolerance to tissue acceptance. Adv Immunol. 2003; 81: 199-252.

7. Le Discorde M, Moreau P, Sabatier P, Legeais JM, Carosella ED. Expression of HLA-G in human cornea, an immune-privileged tissue. Hum Immunol. 2003; 64: 1039-44.

8. Ito $\mathrm{T}$, Ito $\mathrm{N}$, Saathoff $\mathrm{M}$, Stampachiacchiere $\mathrm{B}$, Bettermann $\mathrm{A}$, Bulfone-Paus S, et al. Immunology of the human nail apparatus: the nail matrix is a site of relative immune privilege. J Invest Dermatol. 2005; 125: 1139-48.

9. Cirulli V, Zalatan J, McMaster M, Prinsen R, Salomon DR, Ricordi C, et al. The class I HLA repertoire of pancreatic islets comprises the nonclassical class Ib antigen HLA-G. Diabetes. 2006; 55: 1214-22.

10. Lila N, Rouas-Freiss N, Dausset J, Carpentier A, Carosella ED. Soluble HLA-G protein secreted by allo-specific CD4+ T cells suppresses the allo-proliferative response: a CD4+ $\mathrm{T}$ cell regulatory mechanism. Proc Natl Acad Sci U S A. 2001; 98: 12150-5.

11. Le Gal FA, Riteau B, Sedlik C, Khalil-Daher I, Menier C, Dausset J, et al. HLA-G-mediated inhibition of antigen-specific cytotoxic T lymphocytes. Int Immunol. 1999; 11: 1351-6.

12. Bainbridge DR, Ellis SA, Sargent IL. HLA-G suppresses proliferation of CD4(+) T-lymphocytes. J Reprod Immunol. 2000; 48: 17-26.

13. Shakhawat A, Shaikly V, Elzatma E, Mavrakos E, Jabeen A, Fernandez $\mathrm{N}$. Interaction between HLA-G and monocyte/macrophages in human pregnancy. J Reprod Immunol. 2010; 85: 40-6.

14. Riteau B, Menier C, Khalil-Daher I, Martinozzi S, Pla M, Dausset J, et al. HLA-G1 co-expression boosts the HLA class I-mediated NK lysis inhibition. Int Immunol. 2001; 13: 193-201.

15. Moreau P, Adrian-Cabestre F, Menier C, Guiard V, Gourand L, Dausset $\mathrm{J}$, et al. IL-10 selectively induces HLA-G expression in human trophoblasts and monocytes. Int Immunol. 1999; 11: 803-11.

16. Roth I, Corry DB, Locksley RM, Abrams JS, Litton MJ, Fisher SJ. Human placental cytotrophoblasts produce the immunosuppressive cytokine interleukin 10. J Exp Med. 1996; 184: 539-48.

17. He J, Tang XF, Chen QY, Mai HQ, Huang ZF, Li J, et al. Ex vivo expansion of tumor-infiltrating lymphocytes from nasopharyngeal carcinoma patients for adoptive immunotherapy. Chin J Cancer. 2012; 31: $287-94$.
18. Yie SM, Yang H, Ye SR, Li K, Dong DD, Lin XM. Expression of human leukocyte antigen G (HLA-G) correlates with poor prognosis in gastric carcinoma. Ann Surg Oncol. 2007; 14: 2721-9.

19. Sebti Y, Le Friec G, Pangault C, Gros F, Drenou B, Guilloux V, et al. Soluble HLA-G molecules are increased in lymphoproliferative disorders. Hum Immunol. 2003; 64: 1093-101.

20. Nuckel H, Rebmann V, Durig J, Duhrsen U, Grosse-Wilde H. HLA-G expression is associated with an unfavorable outcome and immunodeficiency in chronic lymphocytic leukemia. Blood. 2005; 105: 1694-8.

21. Davidson B, Elstrand MB, McMaster MT, Berner A, Kurman RJ, Risberg $B$, et al. HLA-G expression in effusions is a possible marker of tumor susceptibility to chemotherapy in ovarian carcinoma. Gynecol Oncol. 2005; 96: 42-7.

22. Barrier BF, Kendall BS, Sharpe-Timms KL, Kost ER. Characterization of human leukocyte antigen-G (HLA-G) expression in endometrial adenocarcinoma. Gynecol Oncol. 2006; 103: 25-30.

23. Seliger B, Abken H, Ferrone S. HLA-G and MIC expression in tumors and their role in anti-tumor immunity. Trends Immunol. 2003; 24: 82-7.

24. Ibrahim EC, Aractingi S, Allory Y, Borrini F, Dupuy A, Duvillard P, et al. Analysis of HLA antigen expression in benign and malignant melanocytic lesions reveals that upregulation of HLA-G expression correlates with malignant transformation, high inflammatory infiltration and HLA-A1 genotype. Int J Cancer. 2004; 108: 243-50.

25. Lin A, Chen HX, Zhu CC, Zhang X, Xu HH, Zhang JG, et al. Aberrant human leucocyte antigen-G expression and its clinical relevance in hepatocellular carcinoma. J Cell Mol Med. 2010; 14: 2162-71.

26. Yoon BS, Kim YT, Kim JW, Kim SH, Kim JH, Kim SW. Expression of human leukocyte antigen-G and its correlation with interleukin-10 expression in cervical carcinoma. Int J Gynaecol Obstet. 2007; 98: 48-53.

27. Ghandri N, Gabbouj S, Farhat K, Bouaouina N, Abdelaziz H, Nouri A, et al. Association of HLA-G polymorphisms with nasopharyngeal carcinoma risk and clinical outcome. Hum Immunol. 2011; 72: 150-8.

28. Wang HY, Sun BY, Zhu ZH, Chang ET, To KF, Hwang JS, et al. Eight-Signature Classifier for Prediction of Nasopharnyngeal Carcinoma Survival. J Clin Oncol. 2011 ; 29: 4516-25.

29. Cai MY, Zhang B, He WP, Yang GF, Rao HL, Rao ZY, et al. Decreased expression of PinX1 protein is correlated with tumor development and is a new independent poor prognostic factor in ovarian carcinoma. Cancer Sci. 2010; 101: 1543-9.

30. Peng J, Ding T, Zheng LM, Shao JY. [Influence of tumor-associated macrophages on progression and prognosis of nasopharyngeal carcinoma]. Ai Zheng. 2006; 25: 1340-5.

31. Rodriguez JA, Galeano L, Palacios DM, Gomez C, Serrano ML, Bravo MM, et al. Altered HLA class I and HLA-G expression is associated with IL-10 expression in patients with cervical cancer. Pathobiology. 2012; 79: 72-83.

32. Kuper CF, Koornstra PJ, Hameleers DM, Biewenga J, Spit BJ, Duijvestijn AM, et al. The role of nasopharyngeal lymphoid tissue. Immunol Today. 1992; 13: 219-24.

33. Bei JX, Jia WH, Zeng YX. Familial and large-scale case-control studies identify genes associated with nasopharyngeal carcinoma. Semin Cancer Biol. 2012; 22: 96-106.

34. Gonzalez A, Rebmann V, Lemaoult J, Horn PA, Carosella ED, Alegre E. The immunosuppressive molecule HLA-G and its clinical implications. Crit Rev Clin Lab Sci. 2012 ; [Epub ahead of print].

35. Rouas-Freiss N, Moreau P, Ferrone S, Carosella ED. HLA-G proteins in cancer: do they provide tumor cells with an escape mechanism? Cancer Res. 2005; 65: 10139-44.

36. Fujieda S, Lee K, Sunaga H, Tsuzuki H, Ikawa H, Fan GK, et al. Staining of interleukin-10 predicts clinical outcome in patients with nasopharyngeal carcinoma. Cancer. 1999; 85: 1439-45.

37. Ozyar E, Ayhan A, Korcum AF, Atahan IL. Prognostic role of Ebstein-Barr virus latent membrane protein-1 and interleukin-10 expression in patients with nasopharyngeal carcinoma. Cancer Invest. 2004; 22: 483-91.

38. Urosevic M, Kurrer MO, Kamarashev J, Mueller B, Weder W, Burg G, et al. Human leukocyte antigen $G$ up-regulation in lung cancer associates with high-grade histology, human leukocyte antigen class I loss and interleukin-10 production. Am J Pathol. 2001; 159: 817-24. 